OPEN ACCESS

Edited by:

William Small, Jr.,

Loyola University Chicago,

United States

Reviewed by:

Joshua Silverman,

New York University, United States

John E. Mignano,

Tufts University School of Medicine,

United States

*Correspondence:

Christopher P. Cifarelli

cpcifarelli@hsc.wvu.edu

Specialty section: This article was submitted to

Radiation Oncology,

a section of the journal

Frontiers in Oncology

Received: 31 August 2021 Accepted: 14 October 2021 Published: 11 November 2021

Citation:

Cifarelli CP and Jacobson GM (2021) Intraoperative Radiotherapy

in Brain Malignancies: Indications and Outcomes in Primary and

Metastatic Brain Tumors.

Front. Oncol. 11:768168.

doi: 10.3389/fonc. 2021.768168

\section{Intraoperative Radiotherapy in Brain Malignancies: Indications and Outcomes in Primary and Metastatic Brain Tumors}

\author{
Christopher P. Cifarelli ${ }^{1,2 *}$ and Geraldine M. Jacobson ${ }^{2}$ \\ ${ }^{1}$ Department of Neurosurgery, West Virginia University, Morgantown, WV. United States, ${ }^{2}$ Department of Radiation \\ Oncology, West Virginia University, Morgantown, WV, United States
}

Despite the continued controversy over defining an optimal delivery mechanism, the critical role of adjuvant radiation in the management of surgically resected primary and metastatic brain tumors remains one of the universally accepted standards in neurooncology. Local disease control still ranks as a significant predictor of survival in both highgrade glioma and treated intracranial metastases with radiation treatment being essential in maximizing tumor control. As with the emergence and eventual acceptance of cranial stereotactic radiosurgery (SRS) following an era dominated by traditional radiotherapy, evidence to support the use of intraoperative radiotherapy (IORT) in brain tumors requiring surgical intervention continues to accumulate. While the clinical trial strategies in treating glioblastoma with IORT involve delivery of a boost of cavitary radiation prior to the planned standard external beam radiation, the use of IORT in metastatic disease offers the potential for dose escalation to the level needed for definitive adjuvant radiation, eliminating the need for additional episodes of care while providing local control equal or superior to that achieved with SRS in a single fraction. In this review, we explore the contemporary clinical data on IORT in the treatment of brain tumors along with a discussion of the unique dosimetric and radiobiological factors inherent in IORT that could account for favorable outcome data beyond those seen in other techniques.

Keywords: IORT, brain metastases, glioblastoma, radiotherapy, local control, radiobiology

\section{INTRODUCTION}

The utility of adjuvant radiation in the management of high-grade primary central nervous system (CNS) tumors and intracranial metastatic disease is one of the few areas of modern neuro-oncology where consistent consensus opinions exist. Guideline recommendations from neurosurgical, radiation oncology, and medical oncology professional organizations repeatedly acknowledge the advantages in overall survival and progression-free survival times in populations treated with adjuvant radiation following surgical resection where surgery was deemed to be part of the standard of care $(1,2)$. Yet, beyond acceptance under the broad banner of "radiation treatment," significant debates remain with respect to the modality of radiation delivery, dose, fractionation schedule, and time to initiation $(3,4)$. 
With advancements in delivery techniques come the potential for improved outcomes, both in the areas of tumor control data and safety profiles. Over the past several decades, intraoperative radiotherapy (IORT) has emerged as one of those techniques with several studies demonstrating both feasibility and outcome equivalence, if not superiority when applied in the optimal setting. In the current review, we will examine the available clinical data on the use and applicability of IORT in CNS tumors, including metastatic disease and primary tumors. Moreover, we will discuss IORT within the broader context of intracranial brachytherapy, including the use of X-ray, electron, and radioisotope sources, all of which possess advantages and relative disadvantages in dose delivery.

\section{IORT NOMENCLATURE}

In its simplest sense, IORT is defined as a single dose of radiation treatment delivered within the same anesthesia episode of care in which the tumor is resected or biopsied. Beyond this broad definition, several distinct treatment modalities exist under the umbrella of IORT with the primary differentiation between treatments being based on radiation source. A schematic representation of IORT applications is provided in Figure 1.

Intraoperative electron radiotherapy (IOERT) has been widely available and extensively studied in the context of extracranial disease sites (5-7). With its origins in clinical use dating back to the 1960s, IOERT has been demonstrated to be feasible and effective in local control of disease in breast, pancreas, soft tissue sarcomas, head and neck, uterine, and colorectal cancers $(8,9)$. Structural limitations of the applicator tube have restricted use to cavities with clear line-of-sight parameters, although the recently described potential to link IOERT delivery devices to electromagnetic surgical navigation systems could afford access to previously unattainable targets $(10,11)$. Despite decades of use in non-cranial sites, only a single institutional experience has been published describing intracranial use in high-grade glioma (12).

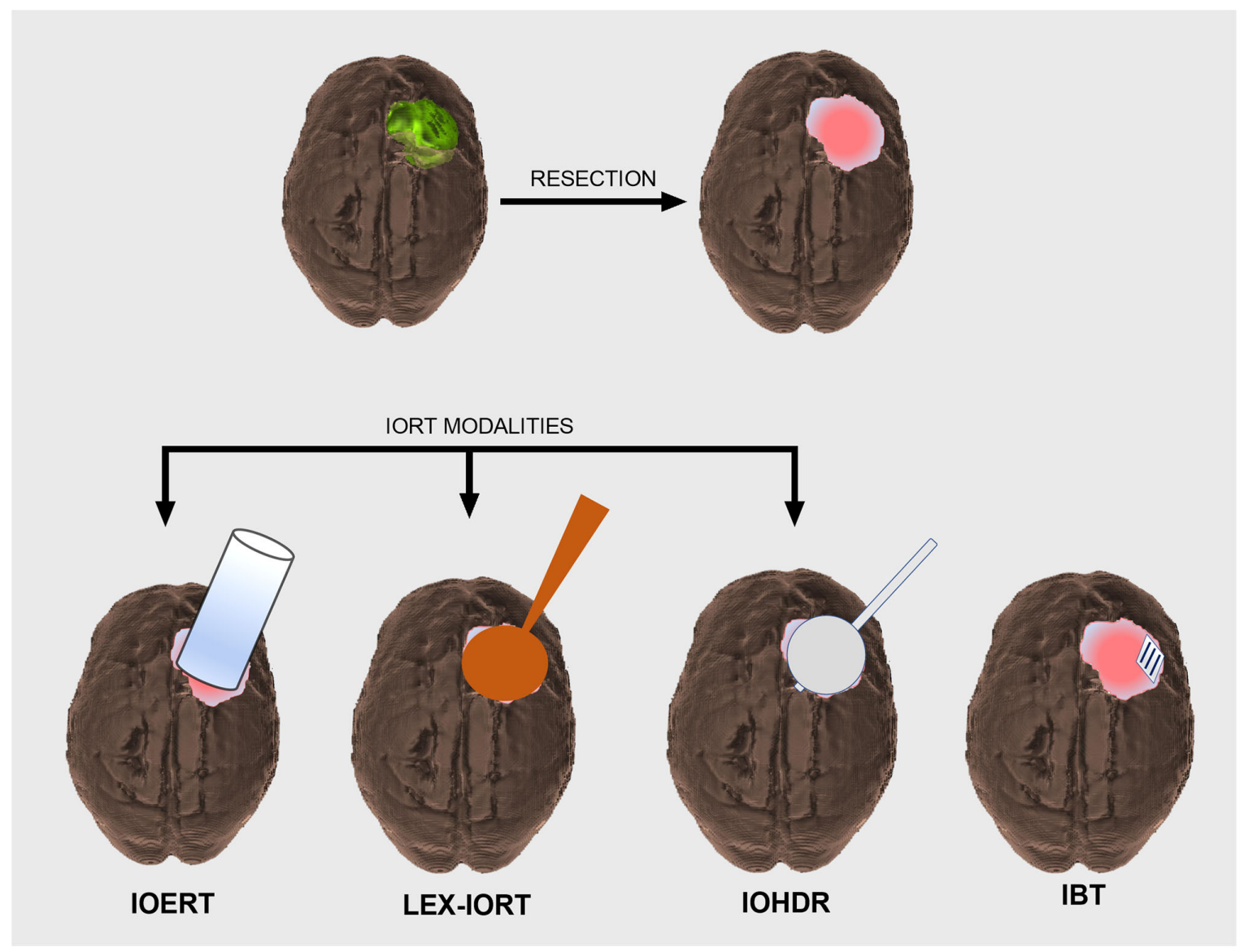

FIGURE 1 | Schematic overview of CNS intraoperative radiotherapy (IORT) modalities. Intracranial tumor (green) prior to surgical resection resulting in a postoperative cavity that will require adjuvant radiation treatment (top row). IORT options include intraoperative electron radiotherapy (IOERT), low-energy X-ray intraoperative radiotherapy (LEX-IORT), and intraoperative high-dose rate brachytherapy (IOHDR) (bottom row). Interstitial brachytherapy (IBT) is distinct from IORT in that radiation dosing extends beyond the anesthetized surgical event. 
Low-energy X-ray intraoperative radiotherapy (LEX-IORT) uses a 30 - to $50-\mathrm{kV}$ isotropic X-ray source with either fixed diameter rigid spherical applicators (Intrabeam ${ }^{\circledR}$, Carl Zeiss Meditec AG, Jena, Germany) or miniaturized X-ray source balloon applicators $\left(\mathrm{Xoft}^{\circledR}\right.$, San Jose, CA, USA). Allowing for a more conformal apposition to the resection cavity walls than the IOERT devices, the LEX-IORT devices exhibit a steep dose gradient with most of the dose delivered within $5-10 \mathrm{~mm}$ of the applicator surface. While the majority of clinical trial and outcome data using LEX-IORT have come from the management of breast cancer via the TARGIT studies, recent clinical trials have involved glioblastoma and more widespread use in surgically resected intracranial metastatic disease $(13,14)$.

Intraoperative high-dose rate brachytherapy (IOHDR), which relies on a sealed radionuclide source being placed within the tumor resection cavity, originated based on the need to treat residual tumor in areas not readily accessible with electrons via an IOERT applicator tube $(15,16)$. Distinct from other brachytherapy methodologies, the entirety of IOHDR treatment is delivered while the patient is anesthetized $(17,18)$. Utilized with regularity in rectal cancer, soft tissue sarcomas, and head and neck carcinomas, no published outcome studies exist detailing the use of IOHDR intracranially. While extensive data do exist with the use of intracranial expandable balloon-based brachytherapy using I-125 and Cs-131, these therapies do not qualify as "intraoperative," nor did data from such studies suggest effectiveness in establishing local control over standard therapy $(19,20)$.

Interstitial brachytherapy (IBT), which is often included in the discussion of IORT, is, in fact, surgically aided radiotherapy rather than intraoperative radiation. Unlike IOERT or LEXIORT, which completes the course of radiation exposure during the single episode of general anesthetic used for tumor resection, IBT delivers the majority of dose to the resection cavity in the days to weeks following implantation via a low-dose rate source material, such as Cs-131. The recent development of a permanent titanium encapsulated Cs-131 implant embedded in a resorbable collagen-based matrix (GammaTile ${ }^{\circledR}$, GT Medical Technologies, Tempe, AZ, USA) has renewed interest in its use for recurrent high-grade tumors of the CNS (21). Clinical trials are currently enrolling patients using these Cs-131 implants under the heading of "surgically targets radiotherapy (STaRT)" (22).

\section{CLINICAL OUTCOME DATA}

\section{Metastatic Disease}

Based on disease incidence and the nearly universal acceptance of the need for adjuvant radiation in brain metastases (BMs) that require surgical removal, the impact of IORT on metastatic disease management has significant potential. Early feasibility and outcome studies of $50 \mathrm{kV}$ LEX-IORT employed the strategy of combining stereotactic biopsy with radiotherapy from a needle-tip applicator (23). The development of spherical applicators and the improved outcomes of patients undergoing gross total resection (GTR) over biopsy only have afforded the opportunity to examine the efficacy of LEX-IORT beyond safety and feasibility (24). In the first such study, Weil et al. provided retrospective analysis of 23 patients diagnosed with solitary brain metastases where $50 \mathrm{kV}$ LEX-IORT was provided at a dose of 14 Gy at $2 \mathrm{~mm}$ depth from the applicator surface. With local control (LC) and distant brain failure (DBF) rate approximately 50\%, LEX-IORT was considered equivalent to alternative strategies of stereotactic radiosurgery (SRS) and whole-brain radiotherapy (WBRT) (25).

Extending from this work, Vargo et al. reported a dose escalation feasibility study where the surface dose was increased to $30 \mathrm{~Gy}$, in line with the available safety data from the Intraoperative Radiotherapy in Newly Diagnosed Glioblastoma (INTRAGO) I trial, where patients received a median dose of $30 \mathrm{~Gy}$ at the applicator surfaces in newly diagnosed glioblastoma followed by an additional $60 \mathrm{~Gy}$ in standard-of-care external beam radiation therapy (EBRT). Developing comparisons between organs at risk (OAR) doses in IORT-treated patients and their theoretical SRS plans to achieve the same cavity margin dose, the authors determined that LEX-IORT was capable of boosting marginal dose beyond traditional SRS techniques (26).

A multi-institutional cooperative study on LEX-IORT in BMs has followed with participations from three international centers also participating the INTRAGO II study for newly diagnosed glioblastoma. In this cohort, 54 patients were treated with a median dose of $30 \mathrm{~Gy}$ at the applicator surface using the Intrabeam $^{\circledR}$ device (Carl Zeiss Meditec AG, Jena, Germany) with reported 1-year LC rate of $88 \%$ and overall survival (OS) of $73 \%$ (14). In comparison, the 1-year LC rate in SRS-treated metastatic surgical resection cavities in a contemporaneous randomized phase III study was only $72 \%$ (27). Subset analysis of these patients revealed an even higher 1-year LC rate of $94 \%$ for GTR versus $62 \%$ for subtotal resection (STR), indicating that the steep dose gradient of LEX-IORT was better suited for the management of microscopic residual disease rather than bulky residual disease. These data are in congruence with the original studies that utilized the needle biopsy approach rather than GTR with 1-year LC rates of $30 \%-50 \%(23,28)$.

An expanded analysis of one of the three institutional cohorts recently provided follow-up with additional patients receiving LEX-IORT for resected BMs with a median marginal dose of 20 Gy. In that study, the 1 -year LC rate was $84 \%$, less than the $88 \%$ LC rate where the median margin dose was $30 \mathrm{~Gy}$, indicating a small but discernable dose dependency on the tumor control rate. Of note, the $20-\mathrm{Gy}$ margin dose study also reported a radiation necrosis (RN) rate of $2.5 \%$ compared with $7 \%$ in the larger study with a median dose of $30 \mathrm{~Gy}$ at the applicator surface (29). In comparison, historical rates of $\mathrm{RN}$ in single fraction radiosurgery treatments based on the $12-\mathrm{Gy}$ volume $\left(V_{12}\right)$ have been shown to be $10 \%$ for a $5-\mathrm{cm}^{3} V_{12}$ (30).

Data from these IORT studies on BMs are summarized in Table 1. 
TABLE 1 | Clinical outcome studies of IORT in brain metastases and glioma.

\begin{tabular}{|c|c|c|c|c|c|c|}
\hline \multicolumn{7}{|c|}{ Metastatic Disease } \\
\hline & $\begin{array}{c}\text { IORT } \\
\text { Modality }\end{array}$ & $\begin{array}{l}\text { Number of } \\
\text { patients }\end{array}$ & Median Dose (Gy) & Local Control Rate & $\begin{array}{l}\text { Additional Adjuvant } \\
\text { Radiation }\end{array}$ & $\begin{array}{l}\text { Overall Survival } \\
\text { (median months) }\end{array}$ \\
\hline Weil et al. (25) & LEX-IORT & 23 & $14(2 \mathrm{~mm})$ & $50 \%(1-y r)$ & $\begin{array}{l}\text { SRS }(n=7) \\
\text { WBRT }(n=6) \\
\text { SRS+WBRT }(n=2)\end{array}$ & 30 \\
\hline Cifarelli et al. (14) & LEX-IORT & 54 & 30 (surface) & $88 \%(1-y r)$ & $\begin{array}{l}\text { SRS }(n=1) \\
\text { WBRT }(n=3)\end{array}$ & na \\
\hline Kahl et al. (29) & LEX-IORT & 44 & 20 (surface) & $84 \%(1-y r)$ & WBRT $(n=10)$ & 26.4 \\
\hline \multicolumn{7}{|c|}{ High Grade Glioma } \\
\hline Schueller et al. (31) & IOERT & 71 & $\begin{array}{c}20 \text { (90-100\% isodose new diagnosis) } \\
25 \text { (90-100\% isodose; recurrent) }\end{array}$ & $4 \%(2-y r)$ & 60 Gy & 12.2 \\
\hline Usychkin et al. (12) & IOERT & 32 & $\begin{array}{l}12.5 \text { (new diagnosis) } \\
10 \text { (recurrent) }\end{array}$ & na & 46-60Gy & $\begin{array}{l}14 \text { (new diagnosis) } \\
10.4 \text { (recurrent) }\end{array}$ \\
\hline Sarria et al. (32) & LEX-IORT & 51 & 30 (surface) & $46 \%(1-y r)$ & 60Gy & 18 \\
\hline
\end{tabular}

LEX-IORT, Iow-energy X-ray IORT; IOERT, intraoperative electron radiotherapy; SRS, stereotactic radiosurgery; WBRT, whole-brain radiotherapy; EBRT, external beam radiotherapy; na, not available.

\section{High-Grade Glioma}

Radiotherapy has been a mainstay of treatment in high-grade glioma (HGG) and glioblastoma (GBM) for decades. Even in the absence of viable chemotherapeutic options based on comorbidities such as age, radiotherapy has remained a critical component of treatment $(33,34)$. In regard to the potential applications of IORT in the management of HGG and GBM, the consensus remains that its utility is in providing a boost to standard-of-care EBRT, usually in the recurrent/progressive disease setting (35). In the largest study of IOERT in HGG, Schueller et al. reported 71 total patients, 52 of which received a 20-Gy boost followed by $60-$ Gy EBRT for newly diagnosed tumors without significant improvement in OS or PFS intervals (31). Usychkin et al. provided an additional description of use of IOERT, both in newly diagnosed and recurrent glioma patients, including anaplastic astrocytoma, GBM, and anaplastic oligodendroglioma based on contemporaneous WHO classifications (12). Of the 32 patients treated, nearly half represented recurrent disease (47\%) with a subtle dose reduction of a median $10 \mathrm{~Gy}$ for recurrent tumors from $12.5 \mathrm{~Gy}$ for newly diagnosed disease. Within the newly diagnosed patients, only six were GBMs with 15 of 17 patients having received postoperative EBRT ranging from 40 to $60 \mathrm{~Gy}$ in addition dose beyond the IOERT (12). Although the median OS for the entire study was 13 months, variability in histological diagnoses and natural disease progression diminishes the overall applicability of the data to HGG patients.

More recently, detailed phase I/II and phase III clinical trials have been designed and implemented for the analysis of IORT impact on newly diagnosed GBM exclusively using LEX-IORT. The INTRAGO clinical trials have established the safe dose range (20-30 Gy) for IORT in newly diagnosed GBM followed by $60-$ Gy EBRT according to standard-of-care treatment regimens $(13,36)$. While the phase III study continues to enroll newly diagnosed patients receiving LEX-IORT plus EBRT versus standard of care EBRT, a pooled analysis of INTRAGO LEX-
IORT-treated patients and similarly treated patients from centers in Peru and China has been published. Representing a total of 51 patients, the median OS at 12,24 , and 36 months was $80 \%, 39 \%$, and $26 \%$, respectively. The overall PFS time was 11.4 months, with $12-, 24-$, and $36-$ month rates of $46 \%, 29 \%$, and $6 \%$, respectively (32). Based on these preliminary findings, the final results of the phase III study (INTRAGO II) have been eagerly anticipated.

Data from these IORT studies on HGG are summarized in Table 1.

\section{DOSIMETRIC DATA}

Given the predominant use of IORT as a means of providing a boost to more traditional external beam radiation treatments, the correct application of dosimetry measures is critical to both safety, in avoidance of adverse radiation effects or RN, and the efficacy of treatment. Dose depth gradients (DDG), homogeneity indices (HI), and relative biological effectiveness (RBE) have been shown to differ slightly among IORT modalities and even more so in comparison with other forms of radiotherapy and radiosurgery. As such, the characteristics of each should ideally be factored into the choice of modality for a given patient.

The steep dose gradient associated with IORT treatments can be advantageous or detrimental depending on primary treatment objectives. For local control in GTR metastatic tumor cavities, such gradients can provide maximal dose to non-visualized microscopic disease while minimizing risk to adjacent brain parenchyma. In the STR metastatic tumor, rapid dose decrements can allow for under treatment of residual disease, especially when IORT serves as the only adjuvant radiotherapy after surgery. The LC rate differences in GTR versus STR treated with LEX-IORT were clearly demonstrated by Cifarelli et al., with 1-year LC rate of $94 \%$ and $62 \%$, respectively (14). In the situation of residual macroscopic tumor, the slightly greater depth-dose distribution provided by 
IOERT compared with LEX-IORT could prove to be beneficial. Once again, the balance of LC versus adverse effects attributed to radiation injury to normal tissue requires consideration.

The homogeneity of dose delivered also has the capacity to impact outcomes, necessitating some evaluation in the process of selecting an ideal IORT modality. While traditional measures of dose homogeneity are expressed as a function of maximal and minimal doses for a particular tumor volume, the cavitary targets treated in IORT add a layer of complexity to such calculations. In comparing the homogeneity of dose between IOERT and IOHDR, Calvo et al. reported that those delivered via IOERT were more evenly distributed across a given depth than those achieved by IOHDR (37). Beyond IORT modalities, homogeneity comparison between LEX-IORT and SRS has been performed, with evidence supporting superior homogeneity in LEX-IORT for single fraction treatment over that of SRS delivered by the Gamma Knife ${ }^{\circledR}$ (Elekta AB, Stockholm, Sweden) (38).

Perhaps the best predictive measure of clinical outcomes following radiation treatment, regardless of modality, is the RBE. Several studies have examined the RBE of IORT modalities, noting specifically that the linear energy transfer (LET) of LEX-IORT produces more lethal macromolecular damage than IOERT (39$41)$. When tested directly in cultured tumor cell populations, the RBE across IORT, IBT, and EBRT energy sources varied based on cell line, indicating a more complicated radiation-induced cell death model than can be explained by stoichiometric differences in DNA damage (42). One such complicating factor includes the potential for radiation-induced bystander effect (RIBE) to increase the cell death following IORT modalities beyond that which would be predicted. Analysis of tumor cavity wound fluid after IORT in breast cancer patients indicates a persistent capacity to impact tumor cell proliferation, epithelial-mesenchymal transformation (EMT), and transcriptional regulation (43-45). Although representing a drastically different tumor microenvironment than that of the chest wall, the potential of cytokine release and immune modulation within the CNS has been hypothesized to play a role in potential IORT effects beyond radiation-induced DNA damage (46).

\section{PLANNING AND LOGISTIC PARAMETERS}

By its very nature, IORT is a team-based approach to patient care consolidated into a single episode. Coordination of the efforts of the surgical team, radiation oncologist, and physics support is a critical component of success and often a limiting factor when determining the feasibility of IORT as a viable treatment. Perhaps in neurosurgical oncology more than in other surgical oncology disciplines, procedures are often scheduled on an urgent rather than elective basis. Peritumoral edema, mass effect, and neurological symptoms all factor into the need for surgical resection in an expedited manner, leaving a short window for schedule coordination among the team members. Whereas the added benefit of a multidisciplinary approach to care is regularly touted as universal, with respect to IORT planning, this can be viewed as a logistic disadvantage $(47,48)$. The lack of availability based on concurrent clinical obligations by a single member of the team can be a significant factor that needs to be considered in the development of an IORT treatment strategy.

Beyond securing the team needed to successfully deliver an IORT treatment, feasibility needs to be established based on dose limitations for OARs and the ability to position an applicator device in the appropriate target area. Unlike IORT for breast or pelvic organs, the OARs within the CNS are capable of falling within an area of high dose, even with a steep dose gradient. Increased applicator size has been associated with significant increases in doses to the optic apparatus and brainstem in LEX-IORT where the diameter of the spherical applicator in LEX-IORT from the Intrabeam ${ }^{\circledR}$ device exceeds $4 \mathrm{~cm}$ (26). Conversely, too little dose can be provided to the intended target if a close approximation of the applicator wall is lost (49). Residual bleeding and cerebrospinal fluid egress in the plane between the cavity wall and applicator are developments that the surgical team needs to be prepared to correct before deeming IORT to be technically feasible. To this end, not all intracranial tumors requiring surgical resection will be eligible for IORT. Hence, lesions that are amenable to GTR and at adequate distance from OAR structures are ideal candidates, although intraoperative judgment of the multidisciplinary team ultimately dictates IORT use.

One of the more obvious advantages of IORT over postsurgical radiation treatment can include elimination of the interval between surgery and radiotherapy or the time to initiation (TTI). Several groups have specifically identified increases in the TTI with decreased efficacy in attaining LC in brain metastases with SRS $(50,51)$. Potential causative factors for such treatment failures may include repopulation of tumor cells within the resection cavity walls and/or the difficulty of accurate target delineation in a resection cavity that has undergone postoperative remodeling $(52,53)$. In the complete elimination of the TTI and target cavity conformality via direct apposition to the tumor resection margin, IORT modalities may have the capacity to provide better outcomes than delayed radiotherapy approaches.

\section{CONCLUSIONS}

Clinical applications for IORT continue to expand into the realm of neuro-oncology. While several studies have established the feasibility of using IORT as an adjunctive radiation treatment for primary CNS malignancies and metastatic disease, data from well-controlled clinical trials currently underway are likely to establish superiority of one modality relative to other forms of IORT or traditional SRS and EBRT techniques over time. While the unique dosimetry and timing of IORT has potential radiobiological advantages in achieving local control of resected CNS tumors, continued investigation in conjunction with novel systemic treatments will be needed in the future.

\section{AUTHOR CONTRIBUTIONS}

CC and GJ contributed to the composition and editing of the manuscript, including the table and figure. All authors contributed to the article and approved the submitted version. 


\section{REFERENCES}

1. Redjal N, Venteicher AS, Dang D, Sloan A, Kessler RA, Baron RR, et al. Guidelines in the Management of CNS Tumors. J Neurooncol (2021) 151 (3):345-59. doi: 10.1007/s11060-020-03530-8

2. Ziu M, Kim BYS, Jiang W, Ryken T, Olson JJ. The Role of Radiation Therapy in Treatment of Adults With Newly Diagnosed Glioblastoma Multiforme: A Systematic Review and Evidence-Based Clinical Practice Guideline Update. J Neurooncol (2020) 150(2):215-67. doi: 10.1007/s11060-020-03612-7

3. Mehta MP. The Controversy Surrounding the Use of Whole-Brain Radiotherapy in Brain Metastases Patients. Neuro Oncol (2015) 17(7):91923. doi: 10.1093/neuonc/nov089

4. Shen CJ, Lim M, Kleinberg LR. Controversies in the Therapy of Brain Metastases: Shifting Paradigms in an Era of Effective Systemic Therapy and Longer-Term Survivorship. Curr Treat Options Oncol (2016) 17(9):46. doi: 10.1007/s11864-016-0423-3

5. Fastner G, Gaisberger C, Kaiser J, Scherer P, Ciabattoni A, Petoukhova A, et al. ESTRO IORT Task Force/ACROP Recommendations for Intraoperative Radiation Therapy With Electrons (IOERT) in Breast Cancer. Radiother Oncol (2020) 149:150-7. doi: 10.1016/j.radonc.2020.04.059

6. Thomas CR, Merrick HW. Intraoperative Radiation Therapy in the Multimodality Approach to Hepatobiliary Tract Cancer. Surg Oncol Clin N Am (2003) 12(4):979-92. doi: 10.1016/s1055-3207(03)00085-1

7. Cambeiro M, Calvo FA, Aristu JJ, Jimenez MM, San-Julian M, Alcalde J, et al. Salvage Surgery and Radiotherapy Including Intraoperative Electron Radiotherapy in Isolated Locally Recurrent Tumors: Predictors of Outcome. Radiother Oncol (2015) 116(2):316-22. doi: 10.1016/j.radonc.2015.07.009

8. Abe M, Takahashi M. Intraoperative Radiotherapy: The Japanese Experience. Int J Radiat Oncol Biol Phys (1981) 7(7):863-8. doi: 10.1016/0360-3016(81) 90001-8

9. Hensley FW. Present State and Issues in IORT Physics. Radiat Oncol (2017) 12(1):37. doi: 10.1186/s13014-016-0754-z

10. Pascau J. Image-Guided Intraoperative Radiation Therapy: Current Developments and Future Perspectives. Expert Rev Med Devices (2014) 11 (5):431-4. doi: 10.1586/17434440.2014.929494

11. García-Vázquez V, Marinetto E, Santos-Miranda JA, Calvo FA, Desco M, Pascau J. Feasibility of Integrating a Multi-Camera Optical Tracking System in Intra-Operative Electron Radiation Therapy Scenarios. Phys Med Biol (2013) 58(24):8769-82. doi: 10.1088/0031-9155/58/24/8769

12. Usychkin S, Calvo F, dos Santos MA, Samblás J, de Urbina DO, Bustos JC, et al. Intra-Operative Electron Beam Radiotherapy for Newly Diagnosed and Recurrent Malignant Gliomas: Feasibility and Long-Term Outcomes. Clin Transl Oncol (2013) 15(1):33-8. doi: 10.1007/s12094-012-0892-1

13. Giordano FA, Brehmer S, Mürle B, Welzel G, Sperk E, Keller A, et al. Intraoperative Radiotherapy in Newly Diagnosed Glioblastoma (INTRAGO): An Open-Label, Dose-Escalation Phase I/II Trial. Neurosurgery (2019) 84(1):41-9. doi: 10.1093/neuros/nyy018

14. Cifarelli CP, Brehmer S, Vargo JA, Hack JD, Kahl KH, Sarria-Vargas G, et al. Intraoperative Radiotherapy (IORT) for Surgically Resected Brain Metastases: Outcome Analysis of an International Cooperative Study. J Neurooncol (2019) 145(2):391-7. doi: 10.1007/s11060-019-03309-6

15. Nag S, Tippin D, Ruymann FB. Intraoperative High-Dose-Rate Brachytherapy for the Treatment of Pediatric Tumors: The Ohio State University Experience. Int J Radiat Oncol Biol Phys (2001) 51(3):729-35. doi: 10.1016/s0360-3016(01) 01697-2

16. Nag S, Martinez-Monge R, Ruymann FB, Bauer CJ. Feasibility of Intraoperative High-Dose Rate Brachytherapy to Boost Low Dose External Beam Radiation Therapy to Treat Pediatric Soft Tissue Sarcomas. Med Pediatr Oncol (1998) 31(2):79-85. doi: 10.1002/(sici)1096-911x(199808) 31:2<79::aid-mpo6>3.0.co;2-w

17. Harrison LB, Minsky BD, Enker WE, Mychalczak B, Guillem J, Paty PB, et al. High Dose Rate Intraoperative Radiation Therapy (HDR-IORT) as Part of the Management Strategy for Locally Advanced Primary and Recurrent Rectal Cancer. Int J Radiat Oncol Biol Phys (1998) 42(2):325-30. doi: 10.1016/s03603016(98)00211-9

18. Fleming C, Rimner A, Cohen GN, Woo KM, Zhang Z, Rosenzweig KE, et al. High- and Low-Dose-Rate Intraoperative Radiotherapy for Thoracic
Malignancies Resected With Close or Positive Margins. Brachytherapy (2016) 15(2):208-15. doi: 10.1016/j.brachy.2015.11.007

19. Welsh J, Sanan A, Gabayan AJ, Green SB, Lustig R, Burri S, et al. GliaSite Brachytherapy Boost as Part of Initial Treatment of Glioblastoma Multiforme: A Retrospective Multi-Institutional Pilot Study. Int J Radiat Oncol Biol Phys (2007) 68(1):159-65. doi: 10.1016/j.ijrobp.2006.11.053

20. Kleinberg LR, Stieber V, Mikkelsen T, Judy K, Weingart J, Barnett G, et al. Outcome of Adult Brain Tumor Consortium (ABTC) Prospective DoseFinding Trials of I-125 Balloon Brachytherapy in High-Grade Gliomas: Challenges in Clinical Trial Design and Technology Development When MRI Treatment Effect and Recurrence Appear Similar. J Radiat Oncol (2015) 4 (3):235-41. doi: 10.1007/s13566-015-0210-y

21. Nakaji P, Smith K, Youssef E, Thomas T, Pinnaduwage D, Rogers L, et al. Resection and Surgically Targeted Radiation Therapy for the Treatment of Larger Recurrent or Newly Diagnosed Brain Metastasis: Results From a Prospective Trial. Cureus (2020) 12(11):e11570. doi: 10.7759/cureus.11570

22. Weinberg J. Clinical Trials in Progress: ROADS Trial. Oncology (Williston Park) (2021) 35(8):495. doi: 10.46883/ONC.2021.3508.0495

23. Cosgrove GR, Hochberg FH, Zervas NT, Pardo FS, Valenzuela RF, Chapman P. Interstitial Irradiation of Brain Tumors, Using a Miniature Radiosurgery Device: Initial Experience. Neurosurgery (1997) 40(3):518-23; discussion 5235. doi: 10.1097/00006123-199703000-00018

24. Colombo F, Francescon P, Cavedon C, Casentini L, Zanusso M. Employ of a New Device for Intra-Operative Radiotherapy of Intracranial Tumours. Acta Neurochir (Wien) (2001) 143(8):827-31. doi: 10.1007/s007010170037

25. Weil RJ, Mavinkurve GG, Chao ST, Vogelbaum MA, Suh JH, Kolar M, et al. Intraoperative Radiotherapy to Treat Newly Diagnosed Solitary Brain Metastasis: Initial Experience and Long-Term Outcomes. J Neurosurg (2015) 122(4):825-32. doi: 10.3171/2014.11.JNS1449

26. Vargo JA, Sparks KM, Singh R, Jacobson GM, Hack JD, Cifarelli CP Feasibility of Dose Escalation Using Intraoperative Radiotherapy Following Resection of Large Brain Metastases Compared to Post-Operative Stereotactic Radiosurgery. J Neurooncol (2018) 140(2):413-20. doi: 10.1007/s11060-0182968-4

27. Mahajan A, Ahmed S, McAleer MF, Weinberg JS, Li J, Brown P, et al. PostOperative Stereotactic Radiosurgery Versus Observation for Completely Resected Brain Metastases: A Single-Centre, Randomised, Controlled, Phase 3 Trial. Lancet Oncol (2017) 18(8):1040-8. doi: 10.1016/S1470-2045(17) 30414-X

28. Pantazis G, Trippel M, Birg W, Ostertag CB, Nikkhah G. Stereotactic Interstitial Radiosurgery With the Photon Radiosurgery System (PRS) for Metastatic Brain Tumors: A Prospective Single-Center Clinical Trial. Int J Radiat Oncol Biol Phys (2009) 75(5):1392-400. doi: 10.1016/j.ijrobp.2009.01.022

29. Kahl KH, Balagiannis N, Höck M, Schill S, Roushan Z, Shiban E, et al. Intraoperative Radiotherapy With Low-Energy X-Rays After Neurosurgical Resection of Brain Metastases-an Augsburg University Medical Center Experience. Strahlenther Oncol (2021). doi: 10.1007/s00066-021-01831-z

30. Milano MT, Grimm J, Niemierko A, Soltys SG, Moiseenko V, Redmond KJ, et al. Single- and Multifraction Stereotactic Radiosurgery Dose/Volume Tolerances of the Brain. Int J Radiat Oncol Biol Phys (2021) 110(1):68-86. doi: 10.1016/j.ijrobp.2020.08.013

31. Schueller P, Micke O, Palkovic S, Schroeder J, Moustakis C, Bruns F, et al. 12 Years' Experience With Intraoperative Radiotherapy (IORT) of Malignant Gliomas. Strahlenther Oncol (2005) 181(8):500-6. doi: 10.1007/s00066-0051354-2

32. Sarria GR, Sperk E, Han X, Sarria GJ, Wenz F, Brehmer S, et al. Intraoperative Radiotherapy for Glioblastoma: An International Pooled Analysis. Radiother Oncol (2019) 142:162-7. doi: 10.1016/j.radonc.2019.09.023

33. Okada M, Miyake K, Tamiya T. Glioblastoma Treatment in the Elderly. Neurol Med Chir (Tokyo) (2017) 57(12):667-76. doi: 10.2176/nmc.ra.20170009

34. Wick W, Platten M, Meisner C, Felsberg J, Tabatabai G, Simon M, et al Temozolomide Chemotherapy Alone Versus Radiotherapy Alone for Malignant Astrocytoma in the Elderly: The NOA-08 Randomised, Phase 3 Trial. Lancet Oncol (2012) 13(7):707-15. doi: 10.1016/S1470-2045(12)70164-X

35. Giordano FA, Wenz F, Petrecca K. Rationale for Intraoperative Radiotherapy in Glioblastoma. J Neurosurg Sci (2016) 60(3):350-6. 
36. Giordano FA, Brehmer S, Abo-Madyan Y, Welzel G, Sperk E, Keller A, et al. INTRAGO: Intraoperative Radiotherapy in Glioblastoma Multiforme-a Phase I/II Dose Escalation Study. BMC Cancer (2014) 14:992. doi: 10.1186/ 1471-2407-14-992

37. Calvo FA, Meirino RM, Orecchia R. Intraoperative Radiation Therapy First Part: Rationale and Techniques. Crit Rev Oncol Hematol (2006) 59(2):106-15. doi: 10.1016/j.critrevonc.2005.11.004

38. Dahshan BA, Weir JS, Bice RP, Renz P, Cifarelli DT, Poplawski L, et al. Dose Homogeneity Analysis of Adjuvant Radiation Treatment in Surgically Resected Brain Metastases: Comparison of IORT, SRS, and IMRT Indices. Brachytherapy (2021) 20(2):426-32. doi: 10.1016/j.brachy.2020.11.004

39. Shamsabadi R, Baghani HR, Azadegan B, Mowlavi AA. Impact of Spherical Applicator Diameter on Relative Biologic Effectiveness of Low Energy IORT X-Rays: A Hybrid Monte Carlo Study. Phys Med (2020) 80:297-307. doi: 10.1016/j.ejmp.2020.11.018

40. Liu Q, Schneider F, Ma L, Wenz F, Herskind C. Relative Biologic Effectiveness (RBE) of $50 \mathrm{kV}$ X-Rays Measured in a Phantom for Intraoperative Tumor-Bed Irradiation. Int J Radiat Oncol Biol Phys (2013) 85(4):1127-33. doi: 10.1016/ j.ijrobp.2012.08.005

41. Herskind C, Ma L, Liu Q, Zhang B, Schneider F, Veldwijk MR, et al. Biology of High Single Doses of IORT: RBE, 5 R's, and Other Biological Aspects. Radiat Oncol (2017) 12(1):24. doi: 10.1186/s13014-016-0750-3

42. Marthinsen AB, Gisetstad R, Danielsen S, Frengen J, Strickert T, Lundgren S. Relative Biological Effectiveness of Photon Energies Used in Brachytherapy and Intraoperative Radiotherapy Techniques for Two Breast Cancer Cell Lines. Acta Oncol (2010) 49(8):1261-8. doi: 10.3109/0284186X.2010.504226

43. Kulcenty K, Piotrowski I, Rucinski M, Wroblewska JP, Jopek K, Murawa D, et al. Surgical Wound Fluids From Patients With Breast Cancer Reveal Similarities in the Biological Response Induced by Intraoperative Radiation Therapy and the Radiation-Induced Bystander Effect-Transcriptomic Approach. Int J Mol Sci (2020) 21(3):1159. doi: 10.3390/ijms21031159

44. Kulcenty K, Piotrowski I, Zaleska K, Wichtowski M, Wróblewska J, Murawa D, et al. Wound Fluids Collected Postoperatively From Patients With Breast Cancer Induce Epithelial to Mesenchymal Transition But Intraoperative Radiotherapy Impairs This Effect by Activating the Radiation-Induced Bystander Effect. Sci Rep (2019) 9(1):7891. doi: 10.1038/s41598-019-44412-y

45. Piotrowski I, Kulcenty K, Murawa D, Suchorska W. Surgical Wound Fluids From Patients Treated With Intraoperative Radiotherapy Induce Radiobiological Response in Breast Cancer Cells. Med Oncol (2018) 36 (2):14. doi: 10.1007/s12032-018-1243-z

46. Herskind C, Wenz F, Giordano FA. Immunotherapy Combined With Large Fractions of Radiotherapy: Stereotactic Radiosurgery for Brain MetastasesImplications for Intraoperative Radiotherapy After Resection. Front Oncol (2017) 7:147. doi: 10.3389/fonc.2017.00147

47. Palta JR, Biggs PJ, Hazle JD, Huq MS, Dahl RA, Ochran TG, et al. Intraoperative Electron Beam Radiation Therapy: Technique, Dosimetry, and Dose Specification: Report of Task Force 48 of the Radiation Therapy
Committee, American Association of Physicists in Medicine. Int J Radiat Oncol Biol Phys (1995) 33(3):725-46. doi: 10.1016/0360-3016(95)00280-C

48. Beddar AS, Biggs PJ, Chang S, Ezzell GA, Faddegon BA, Hensley FW, et al. Intraoperative Radiation Therapy Using Mobile Electron Linear Accelerators: Report of AAPM Radiation Therapy Committee Task Group No. 72. Med Phys (2006) 33(5):1476-89. doi: 10.1118/1.2194447

49. Sethi A, Emami B, Small W, Thomas TO. Intraoperative Radiotherapy With INTRABEAM: Technical and Dosimetric Considerations. Front Oncol (2018) 8:74. doi: 10.3389/fonc.2018.00074

50. Yusuf MB, Amsbaugh MJ, Burton E, Nelson M, Williams B, Koutourousiou $\mathrm{M}$, et al. Increasing Time to Postoperative Stereotactic Radiation Therapy for Patients With Resected Brain Metastases: Investigating Clinical Outcomes and Identifying Predictors Associated With Time to Initiation. J Neurooncol (2018) 136(3):545-53. doi: 10.1007/s11060-017-2679-2

51. Bander ED, Yuan M, Reiner AS, Panageas KS, Ballangrud Å, Brennan CW, et al. Durable 5-Year Local Control for Resected Brain Metastases With Early Adjuvant SRS: The Effect of Timing on Intended-Field Control. Neurooncol Pract (2021) 8(3):278-89. doi: 10.1093/nop/npab005

52. Soliman H, Ruschin M, Angelov L, Brown PD, Chiang VLS, Kirkpatrick JP, et al. Consensus Contouring Guidelines for Postoperative Completely Resected Cavity Stereotactic Radiosurgery for Brain Metastases. Int J Radiat Oncol Biol Phys (2018) 100(2):436-42. doi: 10.1016/j.ijrobp.2017.09.047

53. McDermott DM, Hack JD, Cifarelli CP, Vargo JA. Tumor Cavity Recurrence After Stereotactic Radiosurgery of Surgically Resected Brain Metastases: Implication of Deviations From Contouring Guidelines. Stereotact Funct Neurosurg (2019) 97(1):24-30. doi: 10.1159/000496156

Conflict of Interest: CC has received speaking honoraria from Carl Zeiss Meditec AG for educational seminars.

The remaining author declares that the research was conducted in the absence of any commercial or financial relationships that could be construed as a potential conflict of interest.

Publisher's Note: All claims expressed in this article are solely those of the authors and do not necessarily represent those of their affiliated organizations, or those of the publisher, the editors and the reviewers. Any product that may be evaluated in this article, or claim that may be made by its manufacturer, is not guaranteed or endorsed by the publisher.

Copyright (C) 2021 Cifarelli and Jacobson. This is an open-access article distributed under the terms of the Creative Commons Attribution License (CC BY). The use, distribution or reproduction in other forums is permitted, provided the original author(s) and the copyright owner(s) are credited and that the original publication in this journal is cited, in accordance with accepted academic practice. No use, distribution or reproduction is permitted which does not comply with these terms. 